\title{
Long-term survival results and prognostic factors of early gastric cancer
}

\author{
BAOJUN HUANG ${ }^{1}$, ZHENNING WANG $^{1}, \mathrm{CHENGZHONG} \mathrm{XING}^{1}, \mathrm{ZHE} \mathrm{SUN}^{1}$, BO ZHAO $^{2}$ and HUIMIAN XU ${ }^{1}$ \\ ${ }^{1}$ Department of Surgical Oncology, First Affiliated Hospital of China Medical University, Shenyang 110001, P.R. China; \\ ${ }^{2}$ Department of Medicine, Saint Barnabas Medical Center, Livingston, NJ 07039, USA
}

Received May 9, 2011; Accepted July 21, 2011

DOI: $10.3892 /$ etm.2011.323

\begin{abstract}
In spite of the favorable prognosis of early gastric cancer (EGC), recurrence or second primary cancers present in certain patients after curative surgery. It is crucial to identify who are at high risk and when. In the present study, 323 patients with EGC who underwent curative surgery were studied. A total of 22 patients $(6.8 \%)$ died of recurrence, 9 patients $(2.8 \%)$ died of a second primary cancer and 65 patients $(20.1 \%)$ died of comorbid diseases during the 0.3-33 years of follow-up. Among the 22 patients with recurrence, hematogenous metastases were noted in over half of the cases $(77.3 \%, 17 / 22)$; of these cases 15 patients had a recurrence within the first decade after surgery. Histological differentiation and nodal status were correlated with recurrence. Among the second primary cancers, remnant gastric, liver, lung and colon were the most common sites, and the second primary cancers primarily occurred in the second decade after surgery (6/9). Multivariate analysis identified nodal status $(\mathrm{HR}=4.20)$, vessel involvement $(\mathrm{HR}=3.40)$ and histological differentiation $(\mathrm{HR}=3.52)$ as independent prognostic factors for disease-free survival. However, gender, age and differentiation were independent influencing factors for overall survival. Comorbid diseases, recurrence and second primary cancers are the main cause of death in EGC patients after curative resection. Thus, treatment of comorbid diseases and a periodic follow-up schedule may contribute to improved prognosis.
\end{abstract}

\section{Introduction}

Early gastric cancer (EGC) is defined as gastric carcinoma confined to the mucosa and/or submucosa irrespective of

Correspondence to: Professor Huimian Xu, Department of Surgical Oncology, First Affiliated Hospital of China Medical University, North Nanjing Street 155, Shenyang 110001, P.R. China E-mail: xuhuimian@126.com

Abbreviations: EGC, early gastric cancer; AGC, advanced gastric cancer; MLN, metastatic lymph node; HR, hazard ratio; CT, computed tomography

Key words: early gastric cancer, prognosis, recurrence, second primary cancer lymph node involvement and tumor size, according to the Japanese Classification of Gastric Carcinoma (JCGC) (1). Compared to advanced gastric cancer (AGC), EGC has a more favorable prognosis after curative resection; the 5-year survival rate is higher than $90 \%$ in Japan (2), Korea (3), and slightly lower in Italy, France and the US (4-6). In spite of the very favorable prognosis of EGC, recurrence or second primary cancers present in certain patients after curative surgery, with the recurrence or second primary cancer rate varying from 1.4 to $13.7 \%(5,7-11)$. It is crucial to understand the prognostic factors and features of recurrence in patients with EGC and to identify who are at high risk and when; a subsequent follow-up schedule or adjuvant therapy may be considered accordingly.

The incidence of EGC is approximately $10 \%$ among in-patients with gastric carcinoma in China. However, due to the relatively low incidence of recurrence and favorable prognosis after curative resection for EGC, relevant studies have been limited. Furthermore, most previous reports have focused on short-term survival results and few have investigated survival over 10 years $(4,5,7,9-11)$. In the present study, we assessed the long-term survival profiles of patients with EGC at our single institute through 0.3-33 years of follow-up. Survival rate, characteristics of recurrence and causes of death were analyzed, and prognostic factors were also discussed, enabling us to provide a more tailored follow-up schedule and treatment for high-risk patients.

\section{Patients and methods}

Between February 1970 and June 2005, a consecutive series of 3,144 gastric cancer patients underwent curative gastrectomy at the Department of Surgical Oncology, First Affiliated Hospital, China Medical University. Among them, 344 patients $(10.9 \%)$ were diagnosed with EGC. The outcome of all of the patients was followed up by outpatient visits, telephone and mail contact and death certificates. At the end of the followup in June 2008, 21 patients were excluded from our study due to incompleted data collection. The rate of follow-up was $96 \%$, and a total of 323 patients were enrolled in the present study after providing informed consent. The median and mean follow-up period for the survivors was 9 and 10.6 years (range 0.3-33), respectively. The loss of follow-up cases and deaths from any other causes other than cancer were treated as censored data for the analysis of disease-free survival. Only 
Table I. Time and patterns of recurrence in 22 patients with EGC.

\begin{tabular}{|c|c|c|c|c|c|c|c|c|c|}
\hline Time & Liver & Lung & Brain & Bone & $\begin{array}{l}\text { Lymph } \\
\text { node }\end{array}$ & $\begin{array}{c}\text { Peritoneal } \\
\text { dissemination }\end{array}$ & Liver/lung & $\begin{array}{l}\text { Liver/lung/ } \\
\text { bone }\end{array}$ & Total \\
\hline$\leq 5$ years & 2 & 2 & 1 & 1 & 0 & 0 & 1 & 1 & 8 \\
\hline$>5$ to $\leq 10$ years & 1 & 1 & 0 & 0 & 1 & 3 & 5 & 0 & 11 \\
\hline$>10$ years & 1 & 1 & 0 & 0 & 1 & 0 & 0 & 0 & 3 \\
\hline Total & 4 & 4 & 1 & 1 & 2 & 3 & 6 & 1 & 22 \\
\hline
\end{tabular}

patients who died of gastric cancer or second primary cancer were considered as tumor-related deaths.

The pattern of recurrence and second primary cancer were confirmed by physical findings, computed tomography (CT), abdominal ultrasonography, bone scan, endoscopy, needle biopsy or surgery, respectively. Each second primary cancer was geographically separate, distinct and consisted of a single lesion to adequately exclude the possibility that the second tumor represented metastasis. In addition, occurrence of a second tumor was at least 6 months after resection of EGC (12). The study protocol was approved by the Ethics Committee of the China Medical University.

Statistical analysis. Data were analyzed by using the SPSS statistical software program (SPSS Inc., Chicago, IL, USA). The Cox proportional hazards regression model was applied to identify prognostic factors. Disease-free and overall survival rates were calculated using Kaplan-Meier estimation and the log-rank test. The correlation between hematogenous metastasis and clinicopathological factors was evaluated by using logistic regression. $\mathrm{p}<0.05$ was considered statistically significant.

\section{Results}

Clinicopathological features. The age of the patients (mean \pm SD) was $54.2 \pm 12$ years (range 19-80); 49 patients were $\leq 40$ years of age, 242 patients were $41-69$ years and 32 patients were $\geq 70$ years. More men than women ( 236 men vs. 87 women) participated in the study. Carcinoma was located in the lower third of the stomach $(\mathrm{L} / \mathrm{LM})$ in 240 patients, in the middle third (M/ML/MU) in 62 patients, in the upper third (U/UM) in 13 patients and in the whole stomach (UML) in 8 patients. Lymphadenectomy was executed based on the JCGC. D1 and/ or D1 plus no. 7, $8 \mathrm{a}, 9$ lymph node dissection was performed in 98 patients, D2 lymphadenectomy in 154 patients and more than D2 lymphadenectomy in 71 patients.

Mucosa carcinoma was diagnosed in 138 patients (42.7\%) and submucosa carcinoma in 185 (57.3\%), based on the depth of tumor invasion. Protruded type (I a and II a) was found in 26 patients (8\%), flat type (II b) in 20 (6.2\%), depressed type (II c and III) in $238(73.7 \%)$ and mixed type in 39 patients (12.1\%), respectively. The tumor diameter ranged from 0.5 to $18 \mathrm{~cm}(3.4 \pm 2.0) ; \leq 1 \mathrm{~cm}$ in 24 patients $(7.4 \%), 1-3 \mathrm{~cm}$ in 167 patients (51.7\%), $3-6 \mathrm{~cm}$ in 93 patients (28.8\%) and $\geq 6 \mathrm{~cm}$ in 39 patients (12.1\%). Well and/or moderately differentiated tumors were found in 117 patients (36.2\%) and poorly differentiated tumors in 206 patients (63.8\%). Vessel involvement occurred in 25 patients $(7.7 \%)$. Lymph node metastasis was detected in 51 cases (15.8\%) and the median number of MLNs
Table II. Site distribution and occurrence of second primary cancers in 9 patients with EGC.

\begin{tabular}{lccc}
\hline $\begin{array}{l}\text { Second primary } \\
\text { cancers }\end{array}$ & \multicolumn{3}{c}{$\begin{array}{c}\text { Time to occurrence } \\
\text { (years) }\end{array}$} \\
\cline { 2 - 4 } & $\leq 5$ & $>5$ and $\leq 10$ & $>10$ \\
\hline Liver $(n=2)$ & & & 2 \\
Lung $(n=2)$ & & 1 & 1 \\
Colon $(n=1)$ & 1 & & 1 \\
Ureter $(n=1)$ & & 1 & 2 \\
Remnant stomach $(n=3)$ & & & \\
\hline
\end{tabular}

was 2 (range 1-16). The clinicopathological terminology in this study follows the JCGC.

Survival rate, cause and time of death. The 5-, 10-, 15- and 20-year disease-free survival rates in the patients were 97.0, $91.3,87.1$ and $82.5 \%$, respectively, while the overall survival rates for the different periods above were 93.9, 80.7, 65.5 and $45.9 \%$, respectively.

A total of 22 patients (6.8\%) died of recurrence, 9 patients (2.8\%) died of second primary cancers and 65 patients (20.1\%) died of comorbid diseases, such as ischemic heart disease, cerebrovascular disease, respiratory disease, hepatic cirrhosis and accidents, throughout the 0.3-33 years of follow-up. Among the relapsed cases, hematogenous metastasis was the major pattern of recurrence $(77.3 \%, 17 / 22)$, often presenting in the lung, liver, brain and bone marrow. Peritoneal dissemination and lymph node metastases were also noted in 3 and 2 patients, respectively. Notably, 15 out of 17 hematogenous metastases occurred within the first decade and 8 metastases occurred within the first 5 years after surgery. Recurrence occurring in the first decade accounted for $86.4 \%$ (19/22), the earliest case presented itself as brain metastasis 1 year after surgery. The time and patterns of recurrence are summarized in Table I.

The site distribution and time to occurrence of second primary cancers are shown in Table II. Most second primary cancers (6/9) developed 10 years after the original treatment and often presented in the remnant stomach, liver, lung and colon. The latest case happened at 30 years after surgery as a remnant gastric cancer. There was no significant clinicopathological factor associated with second primary cancer through univariate and multivariate analysis (data not shown).

Prognostic factors for disease-free survival in early gastric cancer. Univariate analysis of the ten potential prognostic 
Table III. Univariate analysis of the prognosis of patients with EGC.

\begin{tabular}{|c|c|c|c|c|c|c|c|}
\hline \multirow[b]{2}{*}{ Factors } & \multicolumn{7}{|c|}{ Disease-free survival rate (\%) } \\
\hline & Total & Deceased & 5-year & 10-year & 15 -year & 20-year & P-value \\
\hline \multicolumn{8}{|l|}{ Gender } \\
\hline Male & 236 & 21 & 97.3 & 92.1 & 86.5 & 84.5 & \multirow[t]{2}{*}{0.3270} \\
\hline Female & 87 & 10 & 96.3 & 89.1 & 89.1 & 69.6 & \\
\hline \multicolumn{8}{|l|}{ Age (years) } \\
\hline$\leq 40$ & 49 & 4 & 95.5 & 92.8 & 87.4 & - & \multirow[t]{3}{*}{$0.0460^{\mathrm{a}}$} \\
\hline $41-69$ & 242 & 22 & 98.3 & 92.0 & 88.8 & 83.2 & \\
\hline$\geq 70$ & 32 & 5 & 88.9 & 83.9 & 67.1 & - & \\
\hline \multicolumn{8}{|l|}{ Site } \\
\hline L/LM & 240 & 23 & 96.9 & 90.2 & 85.0 & 83.3 & \multirow[t]{4}{*}{0.7650} \\
\hline $\mathrm{M} / \mathrm{ML} / \mathrm{MU}$ & 62 & 7 & 96.7 & 93.7 & 90.4 & 73.9 & \\
\hline U/UM & 13 & 1 & 100.0 & 91.7 & - & - & \\
\hline UML & 7 & 0 & - & - & - & - & \\
\hline \multicolumn{8}{|l|}{ Tumor size $(\mathrm{cm})$} \\
\hline$\leq 1.0$ & 24 & 2 & 100.0 & 94.4 & 80.9 & - & \multirow[t]{4}{*}{0.5700} \\
\hline $1.1-3.0$ & 167 & 14 & 96.1 & 92.0 & 86.5 & 83.9 & \\
\hline $3.1-5.9$ & 93 & 13 & 97.7 & 88.3 & 86.2 & 75.5 & \\
\hline$\geq 6$ & 39 & 2 & 97.4 & 93.8 & - & - & \\
\hline \multicolumn{8}{|c|}{ Depth of invasion } \\
\hline Mucosal & 138 & 11 & 97.8 & 94.9 & 89.9 & 83.9 & \multirow[t]{2}{*}{0.1990} \\
\hline Submucosal & 185 & 20 & 96.5 & 88.2 & 84.9 & 82.4 & \\
\hline \multicolumn{8}{|l|}{ Differentiation } \\
\hline Well/moderate & 117 & 19 & 93.7 & 85.8 & 77.5 & - & \multirow[t]{2}{*}{$0.0090^{2}$} \\
\hline Poor & 206 & 12 & 98.9 & 94.6 & 93.3 & 85.3 & \\
\hline \multicolumn{8}{|c|}{ Macroscopic type } \\
\hline Protruded & 26 & 4 & 100.0 & 86.5 & - & - & \multirow{4}{*}{0.7460} \\
\hline Flat & 20 & 3 & 100.0 & 87.5 & 87.5 & 77.7 & \\
\hline Depressed & 238 & 22 & 96.4 & 92.1 & 85.6 & 80.2 & \\
\hline Mixed & 39 & 2 & 97.4 & 93.8 & - & - & \\
\hline \multicolumn{8}{|c|}{ Vessel involvement } \\
\hline Negative & 298 & 24 & 97.2 & 92.9 & 89.3 & 86.1 & \multirow[t]{2}{*}{$0.0003^{\mathrm{a}}$} \\
\hline Positive & 25 & 7 & 95.2 & 69.8 & 59.8 & 44.9 & \\
\hline \multicolumn{8}{|c|}{ Lymph node metastasis } \\
\hline Negative & 272 & 19 & 96.8 & 93.8 & 90.7 & 85.2 & \multirow[t]{2}{*}{$0.0006^{\circ}$} \\
\hline Positive & 51 & 12 & 97.9 & 79.7 & 69.7 & - & \\
\hline \multicolumn{8}{|c|}{ Scope of lymph node dissection } \\
\hline D1/D1+ & 98 & 11 & 97.5 & 87.7 & 82.6 & 74.3 & \multirow[t]{3}{*}{0.6590} \\
\hline D2 & 154 & 12 & 95.9 & 93.5 & 89.5 & 86.1 & \\
\hline D2+/D3/D3+ & 71 & 8 & 98.6 & 91.1 & 87.9 & 83.9 & \\
\hline
\end{tabular}

aSignificant difference.

factors was carried out, and the results are summarized in Table III. Significantly worse prognoses were found in patients with good/moderate differentiation, vessel involvement, lymph node metastasis and older age compared to their counterparts, by univariate analysis $(\mathrm{p}<0.05)$. There was no significant difference among other factors on prognosis ( $p>0.05)$.

Only differentiation, vessel involvement and nodal status were identified as independent prognostic factors using Cox regression analysis. Worse survival was observed for patients with well/moderately differentiated tumors than with poorly differentiated tumors ( $\mathrm{HR}=3.52 \mathrm{vs} .1)$, for patients with positive vessel involvement than with negative involvement $(\mathrm{HR}=3.40$ vs. 1), and with lymph node metastasis than without metastasis (HR=4.20 vs. 1) (Table IV). Age was excluded by multivariate analysis. The survival curves stratified according to different clinicopathological factors are shown in Figs. 1-3. 
Table IV. Multivariate analysis of prognostic factors for disease-free survival in ECG. ${ }^{a}$

\begin{tabular}{lccrrrrr}
\hline Factors & \multirow{2}{*}{$\begin{array}{l}\text {-coefficient } \\
\end{array}$} & SE & Wald & P-value & HR & \multicolumn{2}{c}{$95 \%$ CI for HR } \\
\cline { 3 - 8 } & & & & & & Lower & Upper \\
\hline Differentiation & 1.26 & 0.40 & 10.01 & 0.0020 & 3.52 & 1.61 & 7.66 \\
Vessel involvement & 1.22 & 0.44 & 7.91 & 0.0050 & 3.40 & 1.45 & 7.98 \\
Lymph node metastasis & 1.44 & 0.40 & 12.89 & $<0.0001$ & 4.20 & 1.92 & 9.20 \\
\hline
\end{tabular}

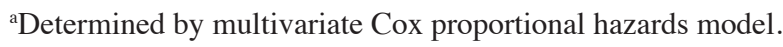

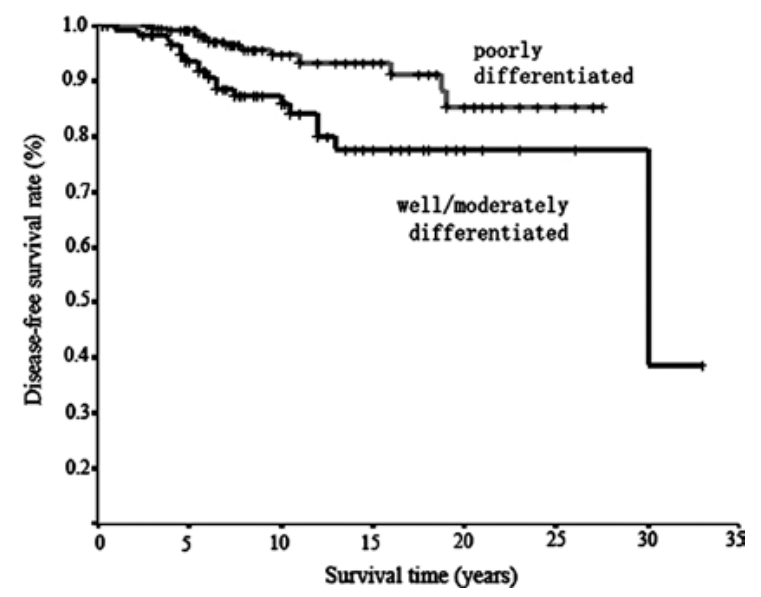

Figure 1. Survival curves in relation to differentiation by Kaplan-Meier estimation. Worse survival was observed in well and/or moderately differentiated cancer than in poorly differentiated cancer; the difference was significant $(\mathrm{p}=0.009)$ using the log-rank test.

Prognostic factors for overall survival in early gastric cancer. Among the ten clinicopathological factors, only gender, age and differentiation were independent influencing factors for overall survival in patients with EGC using multivariate analysis. The HR for death was significantly higher in males than in females, in elders than in younger patients, and in patients with well/moderately differentiated tumors than in those with poorly differentiated ones $(\mathrm{p}<0.05)$. Worse overall survival was also observed with an increase in tumor size, but this was not statistically significant $(\mathrm{p}=0.071)$ (Table V).

Influencing factors for hematogenous recurrence of early gastric cancer. As hematogenous metastasis was the predominant recurrence pattern, its influencing factors were further investigated. Differentiation and nodal status were identified by using multivariate analysis. When the relative risks for recurrence after gastrectomy were set as 1 for patients with poorly differentiated tumors and without lymph node metastasis, the HR of hematogenous metastasis increased to 3.65 $(1.27-10.51, \mathrm{p}=0.016)$ in patients with well/moderately differentiated tumors, and increased to $5.66(1.92-16.67, \mathrm{p}=0.002)$ in patients with lymph node metastasis (Table VI).

\section{Discussion}

In the present study, EGC accounted for only $10.9 \%$ of all resectable gastric carcinomas, which is similar to Western

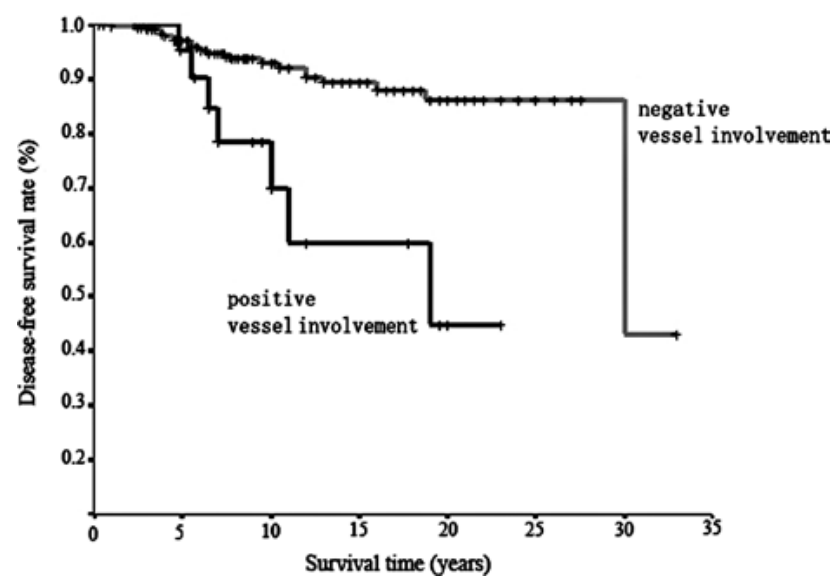

Figure 2. Survival curves in relation to vessel involvement by Kaplan-Meier estimation. Worse survival was observed for positive vessel involvement than for negative vessel involvement; the difference was significant $(p=0.0003)$ using the log-rank test.

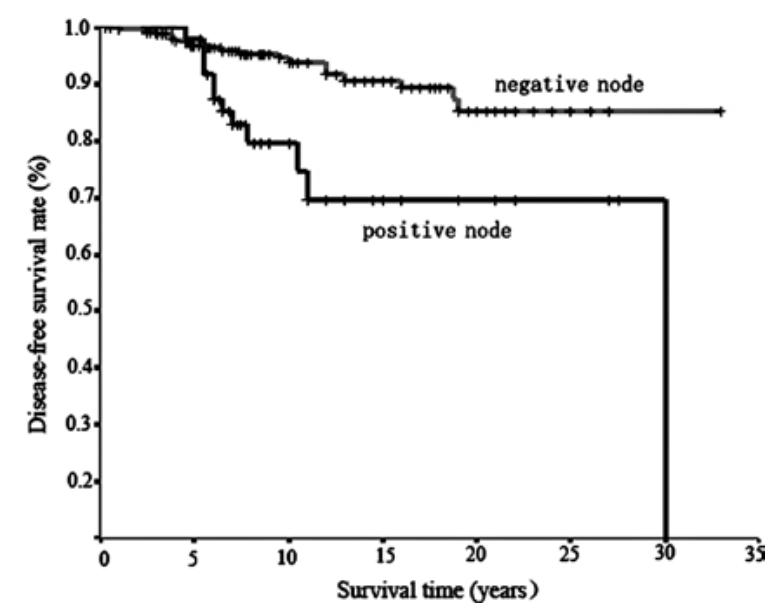

Figure 3. Survival curves in relation to lymph node metastasis by KaplanMeier estimation. Worse survival was observed for cases with positive lymph node metastases than in cases negative for lymph node metastases; the difference was significant $(\mathrm{p}=0.0006)$ using the log-rank test.

countries, but is much lower than Japan and Korea, with an incidence ranging from 40 to $60 \%(7,10,13-17)$. However, the 10 -year disease-free survival rate was $91.3 \%$, suggesting a good prognosis for patients treated at our institute, which closely resembles studies from Japan and Korea. 
Table V. Multivariate analysis of prognostic factors for overall survival in ECG. ${ }^{\text {a }}$

\begin{tabular}{|c|c|c|c|c|c|c|c|}
\hline \multirow[t]{2}{*}{ Factors } & \multirow[t]{2}{*}{$\beta$-coefficient } & \multirow[t]{2}{*}{ SE } & \multirow[t]{2}{*}{ Wald } & \multirow[t]{2}{*}{$\mathrm{P}$-value } & \multirow[t]{2}{*}{$\mathrm{HR}$} & \multicolumn{2}{|c|}{$95 \%$ CI for HR } \\
\hline & & & & & & Lower & Upper \\
\hline Gender & -0.50 & 0.25 & 4.04 & 0.044 & 0.61 & 0.37 & 0.99 \\
\hline Age & 0.05 & 0.01 & 18.18 & $<0.001$ & 1.05 & 1.03 & 1.07 \\
\hline Size & 0.08 & 0.04 & 3.25 & 0.071 & 1.08 & 0.99 & 1.18 \\
\hline Differentiation & 0.48 & 0.22 & 4.94 & 0.026 & 1.62 & 1.06 & 2.47 \\
\hline
\end{tabular}

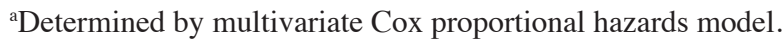

Table VI. Multivariate analysis of factors influencing hematogenous metastasis in ECG. ${ }^{a}$

\begin{tabular}{lccccccr}
\hline Factors & $\beta$-coefficient & SE & Wald & P-value & HR & \multicolumn{2}{c}{$95 \%$ CI for HR } \\
\cline { 3 - 8 } & & & & & & Lower & Upper \\
\hline Differentiation & 1.29 & 0.54 & 5.75 & 0.016 & 3.65 & 1.27 & 10.51 \\
Nodal status & 1.73 & 0.55 & 9.87 & 0.002 & 5.66 & 1.92 & 16.67 \\
\hline
\end{tabular}

a Determined by multivariate logistic regression model.

As indicated by long-term follow-up, recurrence and second primary cancers occurred in 6.8 and $2.8 \%$ of the patients with EGC, respectively, slightly higher than that in Japan and Korea, and lower compared to Western countries $(7-11,18,19)$. Moreover, most recurrence occurred within 10 years after the original surgical treatment $(86.4 \%, 19 / 22)$. Hematogenous spreading was the major pattern of recurrence $(77.3 \%, 17 / 22)$, including 8 cases of early recurrence ( $\leq 5$ years) and 9 cases of late recurrence ( $>5$ years). Our findings corroborate those of Sano et al (9) and Kunisaki et al (10), where hematogenous spreading was also predominant at $65 \%(13 / 20)$ and $71.4 \%$ $(15 / 21)$, respectively. To note, remnant gastric cancer as well as other second primary cancers, including hepatoma and lung cancer, commonly occurred beyond the first decade after surgery, resembling other reports. Yamamoto et al reported that the average interval from surgery to development of a second primary cancer was 7.1 years (18). This may reflect multicenter tumorigenesis. In this study, CT, ultrasonography and endoscopy were the most useful tools for detecting not only recurrence, but also second primary cancers. More than $80 \%$ of recurrences and second primary cancers were detected by $\mathrm{CT}$. Therefore, these tools may be beneficial for early detection of recurrence and second primary cancers; thus a closer follow-up program should be executed.

Since hematogenous metastasis was the main pattern of recurrence, the influencing factors were further discussed in this study. Nodal status was confirmed as an independent risk factor for hematogenous spreading by multivariate analysis. Seven out of 17 patients with hematogenous metastasis had lymph node metastasis and 10 out of 51 patients with lymph node metastasis had vessel invasion, showing a positive correlation between them. This discovery supports the hypothesis that hematogenous recurrence of EGC may be caused by vessel involvement by malignant cells in the mucosal and submucosal layers (20). Histological differentiation status also strongly correlated with hematogenous metastasis $(\mathrm{HR}=3.65)$, with well/moderately differentiated tumors more frequently involved in hematogenous metastasis. This is also supported by studies by Sano et al (9) and Adachi et al (21), where hematogenous metastasis was more frequent in the gastric cancer patients with well-differentiated tumors compared to those with poorly differentiated tumors (68 and 34\%, 41 and $6 \%$, respectively). Although the underlying mechanism of hematogenous metastasis in EGC remains elusive, vessel invasion by cancer cells may be the first step, and histological differentiation may contribute to this course. Further studies based on genomics may elucidate this issue in the future.

Concerning prognostic factors, lymph node metastasis has been well recognized as an important prognostic factor in both EGC and AGC $(22,23)$, which was also confirmed in this study. In addition to nodal status, another two significant prognostic factors identified were histological type and vessel involvement. This may be explained by the fact that patients with well/moderately differentiated tumors and positive nodes are prone to hematogenous metastasis. While many studies have found tumor size and depth of tumor invasion to be significant prognostic factors $(5,10,15,16)$, we did not observe this in our cases. However, tumor size and depth of tumor invasion were slightly correlated with lymph node metastasis (data not shown). Therefore, they may influence prognosis through lymph node metastasis indirectly.

Among the patients who died during the follow-up period, death from comorbid diseases was the most common (20.1\%), followed by recurrence and second primary cancers. With respect to overall survival, age, as well as gender and tumor differentiation, were identified as powerful prognostic indicators in EGC by Cox regression analysis. The rate of death from comorbid diseases increased significantly with age ( $\geq 70$ years). 
In addition, a poorer prognosis was observed in male patients than in female ones. These results were also supported by studies of Kunisaki et al (10) and Bando et al (24). This suggests that patients with curatively resected EGC tend to die of comorbid diseases more frequently at an increased age. Therefore, in order to improve survival and therapeutic outcomes, treatment of comorbid diseases may be quite important, in addition to a periodic follow-up schedule for detecting recurrence and second primary cancers in patients with EGC.

In conclusion, hematogenous recurrence is the major type of relapse in the first decade after surgery, with differentiation and nodal status as independent influencing factors. A second primary cancer commonly occurred in the second decade after surgery. Prognosis is generally worse in EGC patients with vessel involvement, lymph node metastasis or well/moderately differentiated lesions. Death from comorbid diseases was common in patients of increased age. Treatment of comorbid diseases and a periodic follow-up schedule may contribute to the improved prognosis of patients with EGC.

\section{Acknowledgements}

This study was supported by the National Natural Science Foundation of China (no. 30873043 and no. 81071956).

\section{References}

1. Japanese Gastric Cancer Association: Japanese Classification of Gastric Carcinoma. 2nd English edition. Gastric Cancer 1: 10-24, 1998.

2. Shiozawa N, Kodama M, Chida T, Arakawa A, Tur GE and Koyama K: Recurrent death among early gastric cancer patients: 20 years experience. Hepatogastroenterology 41: 244-247, 1994.

3. Kim JP, Hur YS and Yang HK: Lymph node metastasis as a significant prognostic factor in early gastric cancer; analysis of 1,136 early gastric cancers. Ann Surg Oncol 2: 308-313, 1995.

4. Degiuli $M$ and Calvo F: Survival of early gastric cancer in a specialized European center. Which lymphadenectomy is necessary? World J Surg 30: 2193-2203, 2006.

5. Borie F, Rigau V, Fingerhut A, Millat B; French Association for Surgical Research: Prognostic factors for early gastric cancer in France: Cox regression analysis of 332 cases. World J Surg 28: 686-691, 2004

6. Smith JW, Shiu MH, Kelsey L and Brennan MF: Morbidity of radical lymphadenectomy in the curative resection of gastric cancer. Arch Surg 126: 1469-1473, 1991.

7. Lee HJ, Kim YH, Kim WH, Lee KU, Choe KJ, Kim JP and Yang HK: Clinicopathological analysis for recurrence of early gastric cancer. Jpn J Clin Oncol 33: 209-214, 2003.
8. Ikeda Y, Saku M, Kishihara F and Maehara Y: Effective follow-up for recurrence or a second primary cancer in patients with early gastric cancer. Br J Surg 92: 235-239, 2005.

9. Sano T, Sasako M, Kinoshita T and Maruyama K: Recurrence of early gastric cancer. Follow-up of 1475 patients and review of the Japanese literature. Cancer 72: 3174-3178, 1993.

10. Kunisaki C, Akiyama H, Nomura M, et al: Significance of long-term follow-up of early gastric cancer. Ann Surg Oncol 13: 363-369, 2006.

11. Basili G, Nesi G, Barchielli A, Manetti A and Biliotti G: Pathologic features and long-term results in early gastric cancer: report of 116 cases 8-13 years after surgery. World J Surg 27: 149-152, 2003.

12. Moertel CG, Dockerty MB and Baggenstoss AH: Multiple primary malignant neoplasms. III. Tumors of multicentric origin. Cancer 14: 238-248, 1961.

13. Nishi M, Ishihara S, Nakajima T, Ohta K, Ohyama S and Ohta H: Chronological changes of characteristics of early gastric cancer and therapy: experience in the Cancer Institute Hospital of Tokyo, 1950-1994. J Cancer Res Clin Oncol 121: 535-541, 1995.

14. Maehara Y, Kakeji Y, Oda S, Takahashi I, Akazawa K and Sugimachi K: Time trends of surgical treatment and the prognosis for Japanese patients with gastric cancer. Br J Cancer 83: 986-991, 2000.

15. Park IS, Lee YC, Kim WH, Noh SH, Lee KS and Kim H: Clinicopathologic characteristics of early gastric cancer in Korea. Yonsei Med J 41: 607-614, 2000.

16. Hochwald SN, Brennan MF, Klimstra DS, Kim S and Karpeh MS: Is lymphadenectomy necessary for early gastric cancer? Ann Surg Oncol 6: 664-670, 1999.

17. Everett SM and Axon AT: Early gastric cancer in Europe. Gut 41: 142-150, 1997.

18. Yamamoto M, Yamanaka T, Baba H, Kakeji Y and Maehara Y: The postoperative recurrence and the occurrence of second primary carcinomas in patients with early gastric carcinoma. J Surg Oncol 97: 231-235, 2008.

19. Youn HG, An JY, Choi MG, Noh JH, Sohn TS and Kim S: Recurrence after curative resection of early gastric cancer. Ann Surg Oncol 17: 448-454, 2010.

20. Saka M, Katai H, Fukagawa T, Nijjar R and Sano T: Recurrence in early gastric cancer with lymph node metastasis. Gastric Cancer 11: 214-218, 2008.

21. Adachi Y, Yasuda K, Inomata M, Sato K, Shiraishi N and Kitano S: Pathology and prognosis of gastric carcinoma: well versus poorly differentiated type. Cancer 89: 1418-1424, 2000.

22. Yoo CH, Noh SH, Shin DW, Choi SH and Min JS: Recurrence following curative resection for gastric carcinoma. Br J Surg 87: 236-242, 2000.

23. Maehara Y, Emi Y, Baba H, Adachi Y, Akazawa K, Ichiyoshi Y and Sugimachi K: Recurrences and related characteristics of gastric cancer. Br J Cancer 74: 975-979, 1996.

24. Bando E, Kojima N, Kawamura T, Takahashi S, Fukushima N and Yonemura Y: Prognostic value of age and sex in early gastric cancer. Br J Surg 91: 1197-1201, 2004. 